\title{
An IoT based SMART patient health monitoring system
}

\author{
C R Srinivasan, Guru Charan, P Chenchu Sai Babu \\ Department of Instrumentation andn Control Engineeering, Manipal Institute of Technology, \\ Manipal Academy of Higher Education (MAHE), India
}

\begin{tabular}{l}
\hline \hline Article Info \\
\hline Article history: \\
Received Aug 25, 2019 \\
Revised Nov 26, 2019 \\
Accepted Dec 10, 2019
\end{tabular}

Keywords:

Healthcare Internet of Things

IoT

ThingSpeak

Webhooks

\begin{abstract}
Smart and connected health care is of specific significance in the spectrum of applications enabled the Internet of Things (IoT). Networked sensors, either embedded inside our living system or worn on the body, enable to gather rich information regarding our physical and mental health. In specific, the accessibility of information at previously unimagined scales and spatial longitudes combined with the new generation of smart processing algorithms can expedite an advancement in the medical field, from the current post-facto diagnosis and treatment of reactive framework, to an early-stage proactive paradigm for disease prognosis combined with prevention and cure as well as overall administration of well-being rather than ailment. This paper sheds some light on the current methods accessible in the Internet of Things (IoT) domain for healthcare applications. The proposed objective is to design and create a healthcare system centered on Mobile-IoT by collecting patient information from different sensors and alerting both the guardian and the doctor by sending emails and SMS in a timely manner. It remotely monitors the physiological parameters of the patient and diagnoses the illnesses swiftly.
\end{abstract}

Copyright $(2020$ Institute of Advanced Engineering and Science. All rights reserved.

\section{Corresponding Author:}

P Chenchu Sai Babu,

Department of Instrumentation andn Control Engineeering,

Manipal Institute of Technology,

Manipal Academy of Higher Education (MAHE), Manipal, 576104, India.

Email: chenchu.saibabu@manipal.edu

\section{INTRODUCTION}

An essential part of the present world society is the aging population of the world. The average life expectancy has substantially increased as the mortality rate has considerably decreased significantly because of the global advancements in economy, society and healthcare over the past few decades. As a direct outcome, the number of older people across the world has steadily increased. The average percentage of elderly people in the world today (person aged 65 or older) is 7 percent [1]. Furthermore, the percentage of adults over the age of 65 in many nations outpaces the global average, such as 18.5 percent in Finland, 18 percent in Sweden and 15 percent on average for nations in the Organization for Economic Co-operation and Development (OECD) group. This percentage is also expected to increase in the near future. It is anticipated that by the year 2050, 24 percent of the world population of Sweden will be senior citizens, 10 percent of whom will be 80 or more [2].

IoT based technology can deliver a substantial amount of information regarding human, appliances, medical devices, and others. The combination of modern internet technological advancements and IoT provides a great deal of innovative products and services based on wireless communication using low-cost sensors [3]. It offers more collection and processing of data and other services. Any object connecting to IoT demands a unique IP address or mode of identification that can be attained using IPv6.

There exists still several people around the globe whose health is affected by the lack of adequate access to hospitals. Wireless alternatives connected to Internet of things can enable remote monitoring of 
patients rather than visiting the hospital due to the latest wearable technology. A wide range of sensors that are attached to a patient's body could be used to securely obtain patient data, and the data collected can be examined and sent to the main server using various modes of transmission (3G/4G or Wi-Fi) [4]. All doctors have access to the data and can view the data, and decide accordingly on the type of treatment to provide.

People acknowledge that health and well-being is the fundamental condition for promoting the economic development with the passing of time and the development of the society. Most people claim that in terms of time, the current public healthcare system and its support were challenged considerably. Globally, government and the private sector are continuing to invest billions for the development of IoT devices, some of which include the Ministry of Industry and IT's National IoT Plan for China, the European Research Cluster on IoT (IERC), Japan's u-Strategy, UK's Future Internet Initiatives, and Netergit's National Italian Project [5]. Medical and health care IoT applications will benefit the patients significantly by using the finest medical assistance, the fastest treatment time, the most satisfactory service and the lowest medical costs.

Past few years have witnessed a growing value in wearable devices and today numerous devices for personal healthcare, fitness are commercially available [6-8]. Apart from the recreational fitness of existing devices, medical researchers have considered the application of such technology Long-term monitoring, management and medical access of patient's physiological information in remote health surveillance systems for clinical applications [9-12]. Based on existing technological developments, one can easily imagine a time down the road in the future when a 2-3 day duration of periodic physiological monitoring which use low cost wearable sensors precedes your routine physical examination. Over this period, sensors would continuously record signals associated with your physiological parameters and pass that data to a database associated with your medical records. Not only does the doctor have a conventional hospital/Static observations based on laboratory testing of your metabolic and physiological state available for your physical medical examination, but also far richer dynamical record obtained by the set of sensors. The doctor could make much improved health prognosis and advise early intervention, treatment and health decisions that are particularly efficient in improving the quality of your health by using the existing information and decision support systems which provide other people with access to wide range of information on other patients. Such a revolutionary technology can have a groundbreaking effect on global health care systems, drastically reducing costs of healthcare and improving diagnostic speed and accuracy.

This research [13] focuses to link the level of acceptance wearable technology of physicians with that of the users. From the findings, it is evident that $60 \%$ of medical professionals agree to use such devices on the basis of PEU. Considering PEU, 51.41 percent of non-medical personnels agree to use it. This contrast is due to increased level of medical people's exposure and knowledge of these devices.In addition, 72.73 percent and 69.72 percent of medical and non-medical people agree to the adoption of these devices. The study acts as a forum for raising awareness of such wearable devices.

The implemented IoT system provides an overall patient supervision service that includes two key functionalities:

a) Patient location and tracking: accurate patient location knowledge is a useful asset as it enables timely reaction in the event that urgent aid or assistance is required.

b) Patient health status monitoring: The current condition of critical/normal patients should be continually accessible to the doctor or medical staff. Depending on the type of pathology, it may be necessary to collect different pieces of data about the patient's health status (movement, heartbeat, pulse rate, breath, temperature, etc.), possibly automatically detecting abnormal changes in these parameters [14].

The paper is structured as follows: Following study highlights some of the major researched work in this field. In Section II, we highlight the remote healthcare monitoring devices architecture based on wearable sensors, acquiring, analyzing, and visualizing data. In Sections III, together with the conclusions, we highlight the possibilities and difficulties associated with each of these parts.

In the healthcare sector, IoT has now become a daunting technology and needs to pay close attention to its safety aspect. Nonetheless, it's still in its preliminary stages. This is probably due to the lack of technological capacity to support the IoT project and the lack of HKL's effective risk management framework. Therefore, this report suggests HKL to follow the newly updated ISO / IEC 27005:2018, Information Technology - Information Security Risk Management - Security Techniques [15].

Over the past decade, many IoT-based health care applications have been witnessing major developments $[16,17]$. Researchers have been working on an IoT implementation reference model. Jin et al [18]. proposed a standard framework for the development of IoT applications, like smart healthcare. As per this article, the structure of an IoT application is guided by three main points of view: cloud-centered IoT, network-centered IoT and data-centered IoT. All of these three points of view influenced different IoT applications to be implemented.

Most of the proposed remote healthcare and monitoring frameworks leverage three-level architecture: a Wireless Body Area Network (WBAN) which consists of wearable devices equipped 
with sensors for example, data acquisition, networking, communication and service layer [20]. For example [21] offers a system which uses wearable sensors to determine different physiological parameters, including blood pressure, pulse rate and body temperature.

Sensors transmit all the collected information via Bluetooth connection to the gateway server. The gateway server transforms the information into a measurement file and is stored on a remote server for clinicians to later view it over the internet. Using an identical cloud-based storage of medical data, a healthcare monitoring system is addressed in [21] where medical personnel can gain access to the data stored online through the application of content service. WANDA [22] provides a remote monitoring and analysis system for the monitoring of patients having a great danger of heart failure.

Analysis and visualization of the medical data are critical elements of remote health care monitoring systems in relation to the technology of data collection, storage and access. Accurate diagnoses and tracking of the medical condition of the patient are based on long-term analysis of medical data containing different physiological characteristics. Dealing with elevated dimensionality information both in time and quantity makes the task of data assessment quite frustrating, and clinicians are susceptible to error. Though the usage of data collection and visualisation techniques was earlier discussed as an alternative to the abovementioned challenge [23, 24] these techniques have only lately been taken into account in remote healthcare monitoring systems [25].

\section{RESEARCH METHOD}

In this paper, we have designed an IoT-based Health Monitoring System that records the patient's pulse rate and temperature as well as send a mail / Text message alert whenever these readings cross the assigned critical values. ThingSpeak application is used to record pulse-rate and temperature readings so that patient's health could be monitored on the internet from anywhere you are in the world. There is also a panic button attached to allow patients to press it to send mail / SMS to their close relatives and doctor in an emergency. Architecture of IOT based Health Monitoring System as shown in Figure 1.

Our aim is to design a simple, easy to design, and cheap device that serves its purpose in Health Monitoring. We use the following easily available components:

a) Arduino Uno

b) LM35 Temperature Sensor

c) ESP8266 Wi-Fi Module

d) Pulse Rate Sensor

e) Push Button

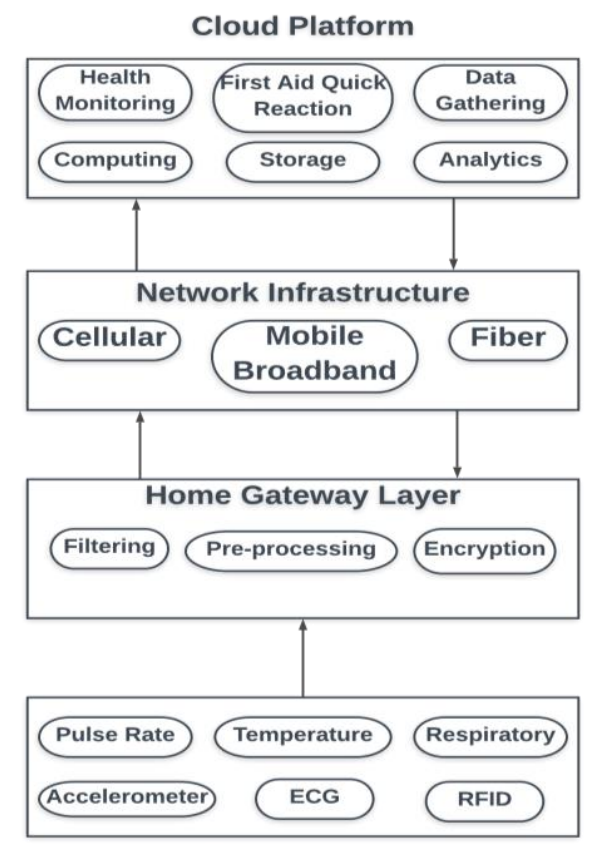

Sensing Layer

Figure 1. Architecture of IOT based Health Monitoring System 


\subsection{Configuration of ThingSpeak}

By using the ThingSpeak website, we are able to monitor the recorded data and control the system via the Internet using ThingSpeak's channels and web pages. ThingSpeak 'Collect' sensor data, 'Analyzes and Visualizes' the data and 'Acts' through a reaction trigger. Using the internet, we used ThingSpeak web page to monitor patient's heartbeat and body temperature online. IFTTT platform is also used to connect ThingSpeak application to the email/SMS service in order to send the alert message whenever the patient is in critical condition. It is necessary to create a Channel in the ThingSpeak application where we can fill the labels to fill the Name of the Channel, Description as required. Create the required amount of fields for the data to be recorded, stored and displayed, in this case, three, for Pulse Rate, Temperature and Panic. Once the Channel is created, the Unique API Key is noted which is the identification tag for this Channel which is connected to the various apps in IFTTT to control the system.

\subsection{Configuration of IFTTT to Record the Data in Google Sheets and Trigger SMS/Mail Based Oon Values on Thingspeak}

If This Then That, also known as IFTTT, is a free internet-based service designed to create basic conditional statement chains, called applets. IFTTT helps to connect various devices and apps together. When some kind of event occurs in any one of the device or apps, IFTTT automatically triggers an action in another app or device. Such programming can be done to any kind of service by creating an applet to link the two services. Here in this project we make use of Webhooks to act as a trigger the SMS or email notification whenever the values recorded in the ThingSpeak application crosses a threshold and when Panic button is triggered. Webhooks is then linked to Gmail and SMS service to trigger the Alert, and it is linked to Google Sheets to record the data in Excel format. Applets can be designed and edited as per the user's requirements. Once the Applets are created a unique URL is generated which is necessary to link ThingSpeak to IFTTT.

\subsection{Thinghttp for Connecting Thingspeak with IFTTT}

The URL from the Webhooks applet of IFTTT is used in the ThingHTTP app of the ThingSpeak and fill the rest of the details about the information required to send to the IFTTT applet, in this case Temperature and Pulse Rate. Once ThingHTTP is created, follow similar steps to create React app for ThingSpeak to trigger the Webhooks on a specific condition. The Condition for Pulse Rate is 160-190 depending on the age of the person while the Condition for Panic is triggered when Panic is 1 or high.

\section{RESULTS AND ANALYSIS}

The aim of this project is to constantly measure, display and monitor the patient's vitals by using a set of sensors which has been successfully implemented. We have also incorporated a mode where a trigger alert is sent to the doctor and the patient's family whenever the vitals reach or cross a particular limit. Our main goal is to design a wearable Smart Device that constantly monitors the vitals. We have successfully implemented that using IoT technology and online applications like ThinkSpeak, Webhooks and IFFFT. As per the required objective, we have successfully designed a device that monitors the patient's vitals and displays it on the ThingSpeak webpage as shown below. All the readings are also displayed in a graphical view for analysis. Each graph displays the live reading where we can check the exact time and location the reading had occurred. All the data recorded are stored in the form of excel sheet in the Google Drive of the maker's google account and can be viewed anytime in the future for analysis. Figure 2 as shown pulse rate and temperature reading.
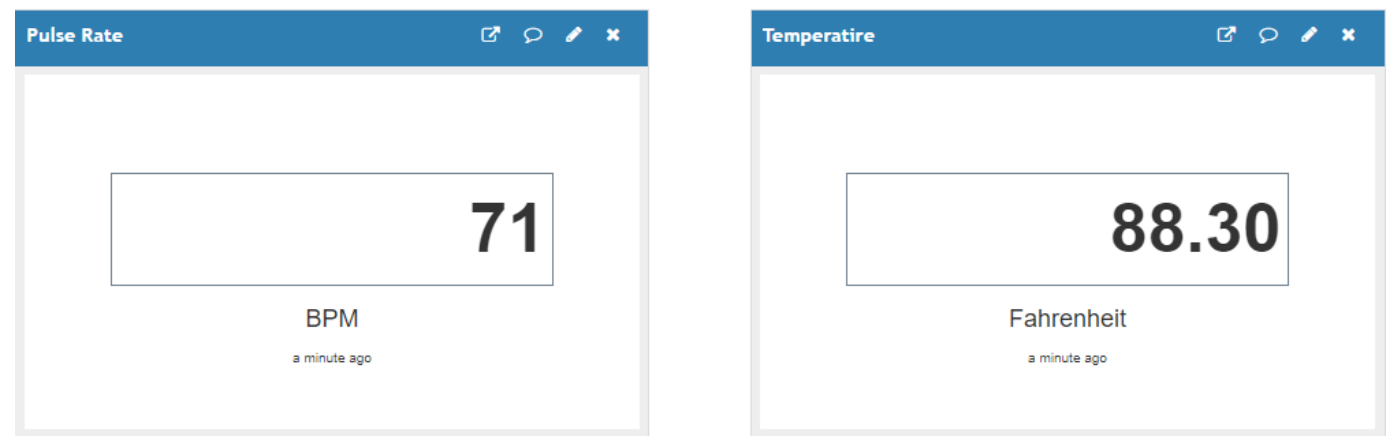

Figure 2. Pulse Rate and Temperature Reading 
All the readings are also displayed in a graphical view for analysis. Each graph displays the live reading where we can check the exact time and location the reading had occurred. Figures 3 and 4 as shown pulse rate, temperature graphical view and panic and location chart.
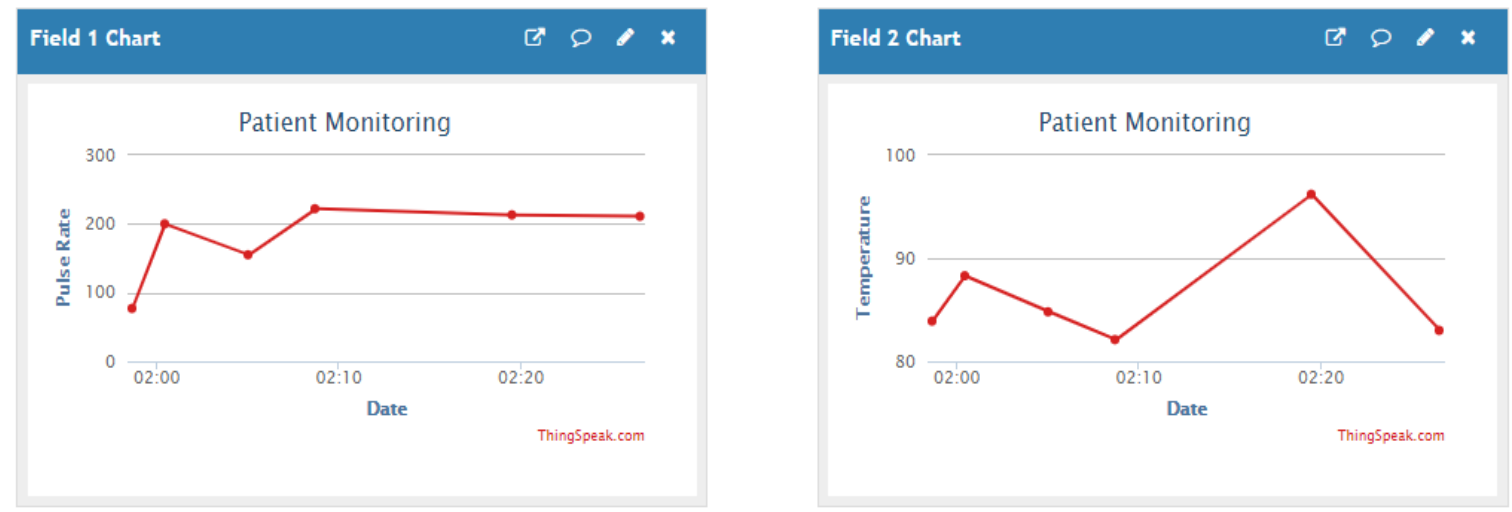

Figure 3. Pulse Rate and Temperature Graphical view
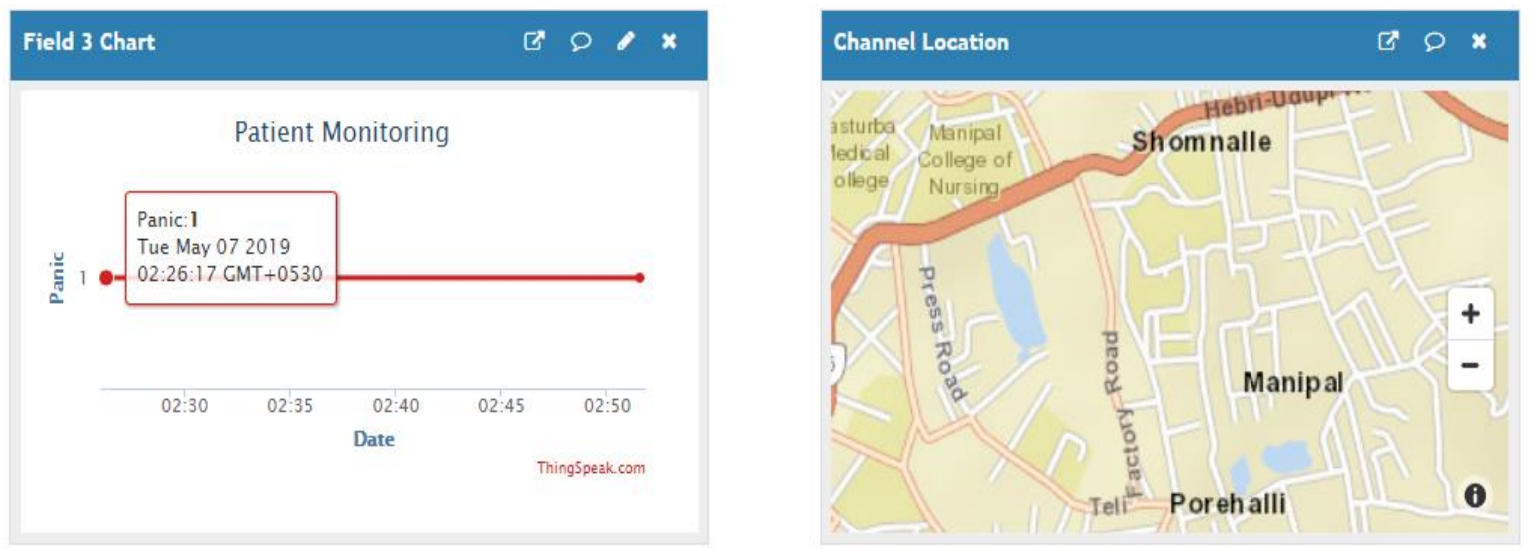

Figure 4. Panic and Location chart

All the data recorded are stored in the form of excel sheet in the Google Drive of the maker's google account and can be viewed anytime in the future for analysis. The excel sheet data as shown in Figure 5. Apart from displaying all the data on the webpage, we have also made provision to monitor the vitals on a mobile-based application called "ThingView Free", available on Play Store. It serves as an easy way to keep track of one's health parameters. We can also know the exact time of every reading.

\begin{tabular}{|c|c|c|c|c|}
\hline A & B & C & D & $E$ \\
\hline created_at & entry_id & Pulse Rate & Temperature & Panic \\
\hline 2019-03-18 16:31:52 UTC & 1 & 154 & 88.4 & \\
\hline 2019-03-18 16:32:27 UTC & 2 & 129 & 79.9 & \\
\hline 2019-03-18 16:32:49 UTC & 3 & 146 & 90.1 & \\
\hline 2019-03-18 16:33:48 UTC & 4 & 114 & 84.6 & \\
\hline 2019-03-18 16:34:46 UTC & 5 & 123 & 82.1 & \\
\hline 2019-03-18 16:35:14 UTC & 6 & 141 & 76.9 & \\
\hline 2019-03-18 16:37:27 UTC & 7 & 114 & 91.1 & \\
\hline 2019-03-18 16:38:56 UTC & 8 & & & \\
\hline 2019-03-18 16:39:50 UTC & 9 & 159 & 86.2 & \\
\hline
\end{tabular}

Figure 5. Excel sheet data 
SMS service has also been successfully implemented where the relatives and the doctor will be able to receive an alert message whenever the Panic button is triggered. Thing view app data and SMS alert as shown in Figures 6 and 7.
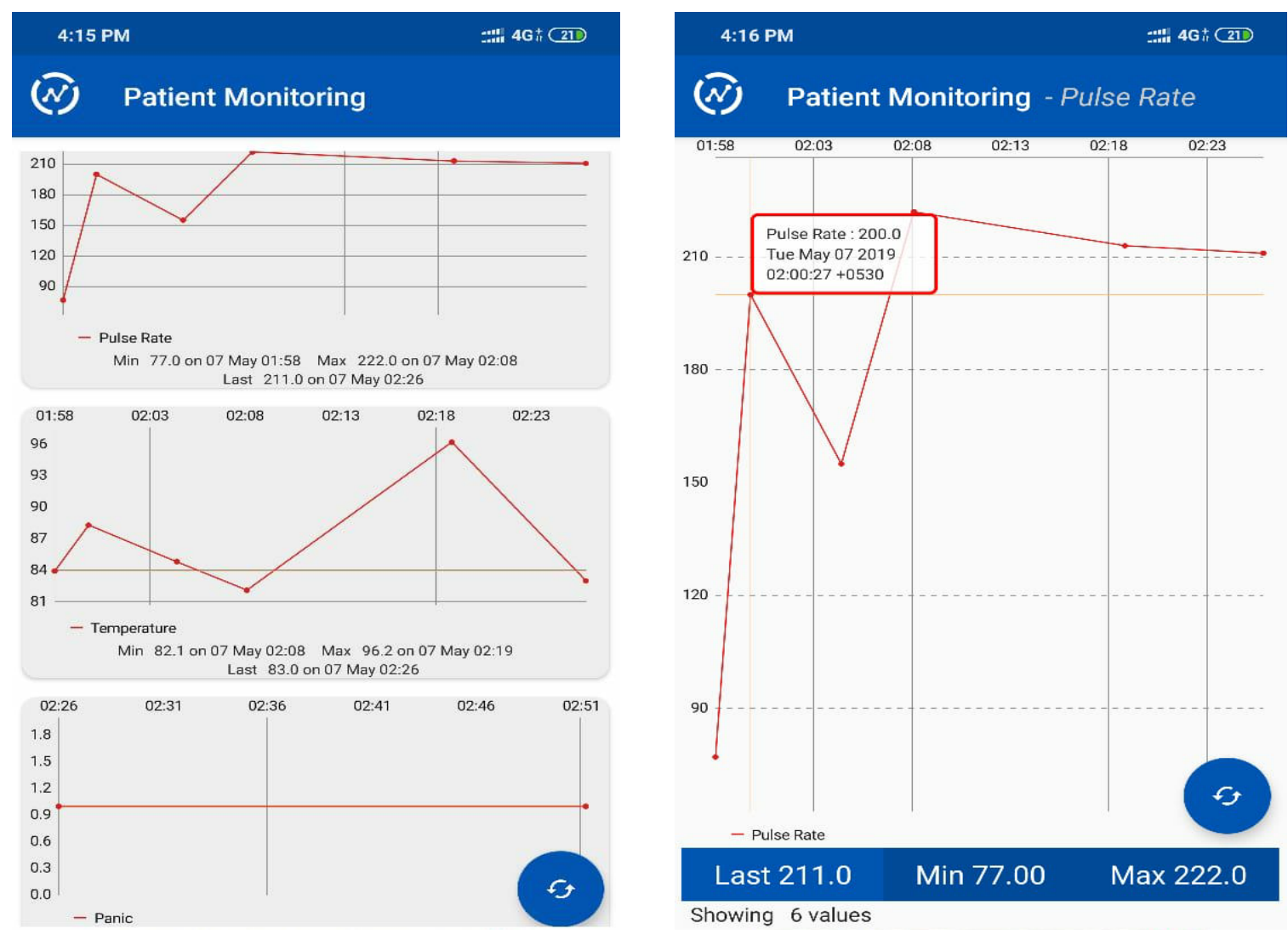

Figure 6. ThingView App data

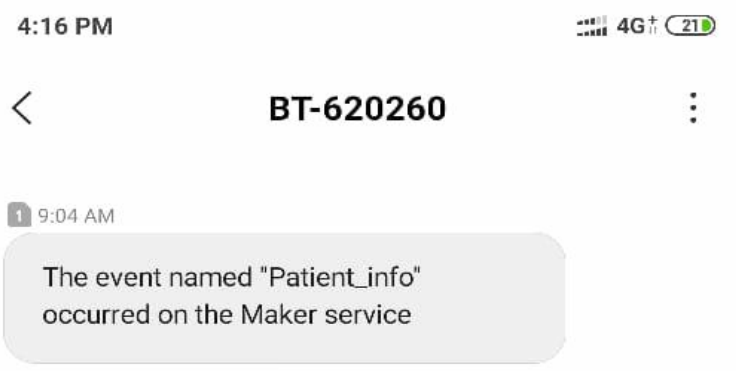

Figure 7. SMS alert

\section{CONCLUSION}

This paper offers a comprehensive survey of recent research trends along with all the smart healthcare opportunities and challenges. There has always been a great demand for needless and costeffective healthcare solutions. With huge funding and increased focus on the smart healthcare arena, users have access to numerous products, services and applications. Since smart healthcare has multifaceted applications it provides researchers a wide range of opportunities to actively innovate innovative products and enhance current architectures. This is primarily due to the need to continually educate and convince healthcare experts to adjust to the digital age. Although IoT-backed smart healthcare technologies can enhance income and enhance quality of lives, if security and safety is compromised the advantages can 
readily be overlooked. Additional measures should be taken to deal with threats and to secure potential information at the ends of both the customer and the developer. Thus, this dynamically increasing industry's vision and long-term achievement lies in the synergy between scientists, healthcare practitioners, and the people.

Apart from that, we can add more sensors like Accelerometer, ECG Sensor, GPS Module, Respiratory sensors, etc., through which we can gain more vital data regarding the patient's health and can result in more precise analysis of their health. Sensors used in this project may not be that accurate. In order to increase the Accuracy of the readings multiple sensors can be used to get an average value of the same reading. With proper analysis and improvements, this device or the mere idea of this project can revolutionize the Healthcare Systems around the globe. There would be no need to personally visit the medical centers for every minor issues for which the Doctor can monitor and suggest ailments from afar. There's also provision for a Panic trigger to alert the relatives about the patient's health which is vital for someone with a hectic life. The device enables Doctors to better manage the use of accessible medical funds and minimize the cost of patient surveillance.

\section{REFERENCES}

[1] Population Reference Bureau, "2014 World Population Data Sheet". [Online]. Available: http://www.prb.org/Publications/Datasheets/2014/2014-worldpopulation- data-sheet.aspx

[2] OECD/European Commission "Sweden-A good life in old age? Monitoring and improving quality in long-term care", June 2013. OECD. [Online]. Available: http://www.oecd.org/els/healthsystems/ Sweden-OECD-EC-GoodTime-in-Old-Age.pdf

[3] F. Yuan Jie, Y. Yue Hong, X. Li Da, Z. Yan, and W. Fan, "IoT-Based Smart Rehabilitation System," Industrial Informatics, IEEE Transactions on, vol. 10, pp. 1568-1577, 2014.

[4] X. Boyi, X. Li Da, C. Hongming, X. Cheng, H. Jingyuan, and B. Fenglin, "Ubiquitous Data Accessing Method in IoT-Based Information System for Emergency Medical Services," Industrial Informatics, IEEE Transactions on, vol. 10, pp. 1578-1586, 2014.

[5] J. Jin, J. Gubbi, S. Marusic, and M. Palaniswami, "An information framework for creating a smart city through Internet of Things," IEEE Internet of Things Journal, vol. 1, pp. 112-121, 2014.

[6] Jawbone Inc., "Jawbone fitness trackers," accessed April 2015. [Online]. Available: https://jawbone.com/up/trackers

[7] FitBit Inc., "flex: Wireless activity + sleep wristband," accessed April 2015. [Online]. Available: https://www.fitbit.com/flex

[8] Apple Inc., “Apple watch,” accessed April 2015. [Online]. Available: https://www.apple.com/watch/

[9] Pantelopoulos and N. Bourbakis, "A survey on wearable sensor-basedsystems for health monitoring and prognosis," IEEE Trans. Sys., Man, and Cybernetics, Part C: Applic. and Reviews, vol. 40, no. 1, pp. 1-12, Jan 2010.

[10] D. Son, J. Lee, S. Qiao, R. Ghaffari, J. Kim, J. E. Lee, C. Song, S. J. Kim, D. J. Lee, S. W. Jun, S. Yang, M. Park, J. Shin, K. Do, M. Lee, K. Kang, C. S. Hwang, N. Lu, T. Hyeon, and D.-H. Kim, "Multifunctional wearable devices for diagnosis and therapy of movement disorders," Nature Nanotechnology, pp. 1-8, 2014.

[11] Page, O. Kocabas, T. Soyata, M. Aktas, and J.-P. Couderc, "Cloud-Based Privacy-Preserving Remote ECG Monitoring and Surveillance," Annals of Noninvasive Electrocardiology (ANEC), 2014. [Online]. Available: http://dx.doi.org/10.1111/anec.12204

[12] R. Paradiso, G. Loriga, and N. Taccini, "A wearable health care system based on knitted integrated sensors," IEEE Trans. Info. Tech. in Biomedicine, vol. 9, no. 3, pp. 337-344, Sept 2005.

[13] Narassima M.S, Anbuudayasankar S.P, Shriram K. Vasudevan, Abhinavaram J, "Physicians' and Users' Perceptions Towards Wearable Health Devices", Indonesian Journal of Electrical Engineering and Computer Science (IJEECS), Vol. 5, No. 1, January 2017, pp. 234-242.

[14] Alessandro Redondi, Marco Chirico, Luca Borsani, Matteo Cesana, Marco Tagliasacchi "An integrated system based on wireless sensor networks for patient monitoring, localization and tracking." Ad Hoc Networks, 11 (2013): 39-53.

[15] Nur Azaliah Abu Bakar, Wan Makhtariah Wan Ramli, and Noor Hafizah Hassan, "The internet of things in healthcare: anoverview, challenges and model plan for security risks management process", Indonesian Journal of Electrical Engineering and Computer Science (IJEECS), Vol. 15, No. 1, July 2019, pp. 414-420.

[16] C. Doukas and I. Maglogiannis, "Bringing IoT and cloud computing towards pervasive healthcare," in Innovative Mobile and Internet Services in Ubiquitous Computing (IMIS), Sixth International Conference on. IEEE, 2012, pp. 922-926.

[17] S. Amendola, R. Lodato, S. Manzari, C. Occhiuzzi, and G. Marrocco, "RFID technology for IoT-based personal healthcare in smart spaces," IEEE Internet of Things Journal, vol. 1, no. 2, pp. 144-152, 2014.

[18] J. Jin, J. Gubbi, S. Marusic, and M. Palaniswami, "An information framework for creating a smart city through internet of things," IEEE Internet of Things Journal, vol. 1, no. 2, pp. 112-121, 2014.

[19] M. Bazzani, D. Conzon, A. Scalera, M. Spirito, and C. Trainito, "Enabling the IoT paradigm in e-health solutions through the VIRTUS middleware," in IEEE 11th Int. Conf. on Trust, Security and Privacy in Computing and Com. (TrustCom), June 2012, pp. 1954-1959. 
[20] Benharref and M. Serhani, "Novel cloud and SOA-based framework for E-Health monitoring using wireless biosensors," IEEE Journal of Biomed. and Health Inf., vol. 18, no. 1, pp. 46-55, Jan 2014.

[21] Rolim, F. Koch, C. Westphall, J. Werner, A. Fracalossi, and G. Salvador, "A cloud computing solution for patient's data collection in health care institutions," in Second Int. Conf. on eHealth, Telemedicine, and Social Medicine, ETELEMED '10., Feb 2010, pp. 95-99.

[22] M. Lan, L. Samy, N. Alshurafa, M.-K. Suh, H. Ghasemzadeh, A. Macabasco-O'Connell, and M. Sarrafzadeh, "Wanda: An end-to-end remote health monitoring and analytics system for heart failure patients," in Proc. of the Conf. on Wireless Health, ser. WH '12. New York, NY, USA: ACM, 2012, pp. 9:1-9:8.

[23] L. Wei, N. Kumar, V. Lolla, E. Keogh, S. Lonardi, C. Ratanamahatana, and H. Van Herle, "A practical tool for visualizing and data mining medical time series," in Proc. 18th IEEE Symposium on Computer-Based Med. Sys., June 2005, pp. 341-346.

[24] Y. Mao, Y. Chen, G. Hackmann, M. Chen, C. Lu, M. Kollef, and T. Bailey, "Medical data mining for early deterioration warning in general hospital wards," in IEEE 11th Int. Conf. on Data Mining Workshops (ICDMW), Dec 2011, pp. 1042-1049.

[25] V. Ukis, S. Tirunellai Rajamani, B. Balachandran, and T. Friese, "Architecture of cloud-based advanced medical image visualization solution," in IEEE Int. Conf. on Cloud Computing in Emerging Markets (CCEM), Oct 2013, pp. 1-5. 